\title{
BATTERY MANAGEMENT OF NEW ENERGY VEHICLE BASED ON CFD
}

\author{
Jia Liu' ${ }^{1}$ Peng-Fei Zhao ${ }^{2}$, Jing Huang ${ }^{3}$ \\ 1,2,3 Department of Mechanical Engineering, Taiyuan Institute of Technology, Shanxi 030008, China \\ ${ }^{1}$ Corresponding author: Chunjun Luo \\ Email: liujiamety222@163.com
}

\begin{abstract}
In order to meet the needs of actual working conditions and people's expectations, electric vehicles using lithium ion batteries as power sources are not completely comparable to traditional fuel vehicles in terms of endurance mileage and driving performance. The main technologies of battery thermal management system are summarized, and the research status of air cooling, liquid cooling and phase change material cooling and heat generation characteristics of batteries are summarized. Based on CFD (Computational Fluid Dynamics) analysis method, the model of liquid cooling and heat dissipation system of batteries is established, and the flow of cold fluid in cooling channel and the temperature change of batteries are analyzed. Based on the simulation results of the original model, the existing problems are analyzed and the cooling passage structure of the cooling system is optimized. The temperature field and flow field of the improved scheme are simulated, analyzed and compared. The results show that, compared with other kinds of batteries, lithium ion batteries have many advantages, such as high voltage, light weight and no pollution. However, the working performance of lithium ion is closely related to the working temperature. When the working temperature is too high, the working safety of lithium ion is not guaranteed; when the working temperature is too low, the chemical reaction rate decreases, and the capacity of lithium ion batteries decreases, which has adverse effects on the endurance mileage and driving performance. Therefore, it is necessary to study the temperature field control of batteries to ensure the normal operation of electric vehicles.
\end{abstract}

Keywords: Lithium Ion Battery; CFD Analysis; Temperature Field; Liquid-Cooled System.

\section{Introduction}

In recent years, China's motor vehicle ownership has maintained high growth. By the end of 2017, China's motor vehicle ownership has reached 310 million [1].

With the increase of motor vehicle ownership, environmental pollution and energy crisis are becoming increasingly prominent. These two problems have also brought great impact to the development of automobile manufacturing in the world. In order to cope with these two problems, governments and automotive enterprises all attach great importance to the research and development of new energy vehicles, and invest a lot of funds and manpower to support the development of new energy automotive products. As one of the most promising new energy vehicles, pure electric vehicle has three core technologies: battery, vehicle control, and motor. Among them, the development and promotion of electric vehicle have been restricted by the problems of battery technology [2].

Lithium ion batteries are the power source of pure electric vehicles, which provide the energy needed in the driving process for pure electric vehicles. The performance of lithium ion batteries directly determines the endurance mileage, power performance, and electric drive capability of pure electric vehicles. Improving the performance of lithium ion batteries is an important content here.

Temperature has an impact on the performance, cycle life, and thermal safety of power batteries. It is necessary to design an efficient thermal management system to control the temperature of power batteries. A new type of Lithium ion battery pack for new energy automobile is studied, and the liquid cooling system of the battery pack is numerically analyzed on the basis of studying the heat generation of the monomer [3].

The temperature field is studied and the structure of liquid cooling and heat dissipation system is established according to its thermal characteristics. An effective and reasonable optimization scheme is proposed for the design model.

The research on temperature field of lithium-ion battery pack and the optimization of thermal management system structure of battery pack can provide reference for the design of battery pack of electric vehicle, which has certain engineering 
application value. The temperature distribution of batteries under different working conditions is calculated by the combination of numerical simulation and experimental verification through the connection of CFD (Computational Fluid Dynamics) and heat transfer, and then the heat transfer structure of batteries is optimized.

Combined with theoretical research, computational simulation and experiments, ANSYS finite element software is used to simulate the temperature field of battery cell and battery pack under different conditions. By optimizing the structure of BTMS, it is ensured that the battery pack can still work at the appropriate temperature under the working conditions of high rate discharge.

Battery thermal management is an indispensable link to ensure the normal running of electric vehicles. Based on the characteristics of lithium-ion batteries, the temperature distribution field of lithium ion batteries in pure electric vehicles is simulated and analyzed, and an effective structure scheme of BTMS is proposed. It provides a reference for the design of the heat dissipation system of battery pack of pure electric vehicle. In order to ensure the heat dissipation requirement, reliability and safety of power battery packs for new energy vehicles, it is significant to develop an effective thermal management system for batteries and design a stable and efficient heat dissipation structure for battery packs to improve the overall performance of batteries.

\section{Current Status and Analysis of Lithium Ion Battery Research at Home and Abroad}

In 1985, Bernadi of the University of California, Berkeley, proposed a model for the heating rate of lithium ion batteries. The model established provides the theoretical basis for the heating rate of batteries, which is widely used in the following years.

The classical model is also used for the calculation of the heating rate of batteries. Gerardine GBotte et al. studied the influence of related parameters (cell density, heat transfer coefficient and current) on the thermal effect of batteries under different conditions of lithium ion batteries using a centralized mass model in 1998 [4].

Noboru Sato et al. directly measured the heating rate q of batteries by experimental method in 2001 . They believed that the heating of batteries consisted of four parts: reaction heat produced by chemical reaction, polarization heat produced by polarization resistance, side reaction heat produced by other side reactions, and Joule heat produced by ohmic internal resistance; Chen et al. used non-stratified simplification to heat batteries in 2005. Thermal radiation is added to the exchange process, and the calculation method of the thermo-coupling model of battery electrochemistry is simplified. Good results have been achieved and adopted many times by researchers. In 2006, Kim et al. took the cylindrical lithium-ion power battery as the research object, established the three-dimensional model of nonstratified battery, and applied the electrochemical thermal coupling model to calculate [5].

Finally, different convective heat transfer coefficients were selected to analyze their effects on the heat transfer of the battery.

In 2008, Rami Sabbah et al., taking a high-power density lithium ion power battery as the research object, compared and analysed the temperature rise of the single battery and the consistency of the temperature between the cells under two different heat dissipation modes of air cooling and phase change material cooling [6].

The final results showed that, when the battery was discharged at high current in the range of 40 $\sim 45^{\circ} \mathrm{C}$, the heat dissipation effect of phase change materials as heat transfer medium for batteries was obviously better than that of the battery directly using air as heat transfer medium.

In 2009, Debashishosh et al. of Ford Company used the method of CFD to analyze the heat dissipation performance of a Ford hybrid electric vehicle battery heat management system, and optimize and improve the geometric model of the original battery heat management system, so as to further enhance the heat dissipation capacity of the battery heat pipe system [7].

As a result, the working environment has been greatly improved. In 2011, Jarrett and other Canadian scholars used CFD method to simulate and analyze the heat dissipation structure of the snakeshaped cooling duct of the battery pack. Finally, the structure of the snake-shaped cooling duct was optimized, and the heat dissipation effect of the battery was significantly improved.

In 2013, Fan et al. carried out CFD simulation analysis on the heat dissipation performance of an air-cooled lithium ion battery module for a hybrid electric vehicle.

The width of the air duct between batteries and the velocity of air flowing through the surface of the battery were studied [8].

Through systematic analysis, the best heat dissipation effect was found between the two factors affecting the heat dissipation performance of the battery.

In 2010, Lin Chengtao, Li Teng and Chen Quanshi of Tsinghua University established a threedimensional thermal model of a $100 \mathrm{Ah}$ lithium ion battery, and carried out simulation calculation and data analysis [9].

It was found that the material and thickness of battery shell had limited influence on the heat dissipation of the battery, which could be simplified in the geometric modeling of the battery and saved a 
lot of calculation time in the simulation experiment. In 2012, Fei Chaohui and Wang Xuelin of Huazhong University of Science and Technology established the thermal characteristic model of single battery, simplified the complex layered structure of battery as an anisotropic material [10].

Through the analysis of the ventilation and heat dissipation scheme of battery box, the improved scheme of ventilation on both sides of battery box and exhaust of intermediate duct was put forward, and the impact of various factors on the temperature field of battery box was studied, which was instructive for the analysis and research of heat dissipation of battery box.

In order to solve the problem of poor heat dissipation effect of lithium ion battery thermal model in 2013, Cheng Yun and Li Jie established a three-dimensional heat dissipation model of lithium ion battery module based on COMSOLMULTIPHYSICS simulation platform, and verified the heat dissipation performance of lithium ion battery module under different heat dissipation modes, which provided a reference for other staff engaged in this research [11]. According to the needs of the project, other staffs can choose the appropriate heat dissipation mode of battery module. Li Junqiu, Wu Puen and Zhang Chengning of Beijing University of Technology carried out research on the design process of battery thermal management and the modeling method of battery thermal analysis in 2014.

The thermal management scheme of battery was designed by forced air cooling and thermistor heating [12]. The correctness of the thermal analysis model and the thermal management scheme of battery were analyzed and verified by simulation and experiment. According to the analysis of the research status at home and abroad, the thermal characteristic model of the battery can be simplified by combining the formulas; the single battery is regarded as an anisotropic material, and its calculation model is measured by classical theoretical model or experiment [13].

There are many studies on the heat dissipation mode of the battery box, on the basis of which more detailed research on the combination of heat dissipation mode is carried out to improve the heat dissipation capacity of the battery box.

Compulsory air-cooled heat dissipation method with air as heat transfer medium has certain advantages: simple heat dissipation structure, low cost of use and later maintenance, etc. its disadvantages are: poor uniformity heat dissipation ability of batteries, excessive temperature difference between single batteries, heat dissipation affected by external temperature, compact structure layout, small heat transfer space, and poor air flow in the battery box for heat dissipation.
When the battery is in the condition of high current charging and discharging, the battery will generate a lot of heat. If the heat dissipation capacity of the battery box is insufficient, the serious problem of the untimely heat dissipation of the battery will occur.

Therefore, the analysis and exploration of the heat dissipation performance of the battery box can better control the maximum temperature of the single battery and the maximum temperature difference of the whole battery module, thereby improving the overall performance of the electric vehicle.

\section{Computational Fluid Dynamics (CFD)}

\subsection{Basic concepts of CFD}

CFD is a systematic analysis method and tool for numerical simulation of fluid flow, heat transfer and related heat transfer phenomena by computer.

Its basic idea can be summed up as follows: replacing the original field of continuous physical quantities in time domain and space domain with the geometry of variable values at a series of finite discrete points, establishing algebraic equations of the relationship between variables at these discrete points through certain principles and methods, and then solving algebraic equations to obtain approximate values of field variables.

\subsection{CFD calculation process}

The calculation process of CFD is mainly divided into three parts: pre-processing, solution and postprocessing. Pre-processing: It takes the longest time in CFD calculation. It mainly carries out model trimming, grid generation and setting of computational domain and boundary conditions. Solution: CFD software mainly uses finite difference method, finite element method, spectral method, and finite volume method.

Post-processing: Simulation data are visually output, such as grid display, contour map, vector map, animation output, etc.

The finite volume method is used to calculate fluid-solid coupling [14].

The equations involved in this method are conservative equations in integral form. Integration is the most commonly used algorithm in computational fluid dynamics for every control body of grid generation.

The flow chart of CFD solution is shown in Figure 1. 


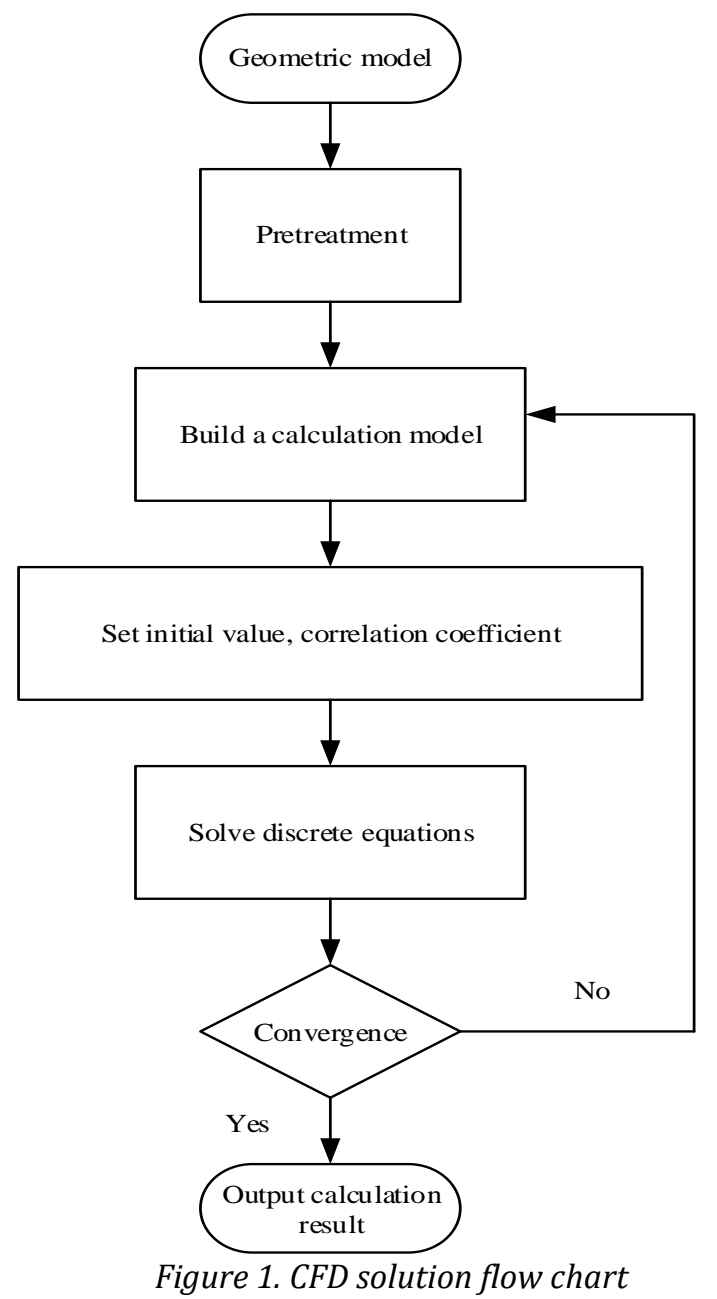

\subsection{Computational equations for CFD}

The flow of any fluid is physically controlled by three laws: the law of mass conservation, Newton's second law, and the law of energy conservation.

The basic governing equations of CFD heat transfer are continuity equation, momentum conservation equation, and energy conservation equation.

The CFD simulation software simulates fluid flow and heat transfer through the above three equations. In addition, when the fluid is turbulent, its flow is extremely complex and needs turbulence equation to calculate.

The continuity equation follows the law of mass conservation, which means that the increment of mass per unit time equals the net mass flowing into the fluid micro-cluster at the same time interval. Its expression is:

$$
\frac{\partial \rho}{\partial \mathrm{t}}+\frac{\partial\left(\rho \mu_{\mathrm{i}}\right)}{\partial \mathrm{x}_{\mathrm{i}}}=0
$$

In Formula (1), $\rho$ indicates the density of the fluid, $\mathrm{t}$ is time, and $\mu_{\mathrm{i}}$ refers to the component of the fluid velocity along the direction $i$.
The momentum equation is based on the law of conservation of momentum. Its meaning is that the volume force acting on the fluid micro-cluster equals the change rate of the momentum of the fluid microcluster with time. Its expression is:

$\frac{\partial}{\partial t}\left(\rho \mu_{u}\right)+\frac{\partial}{\partial x_{j}}\left(\rho \mu_{i} \mu_{j}\right)=-\frac{\partial P}{\partial x_{j}}+\frac{\partial \tau_{i j}}{\partial x_{j}}+\rho g_{i}+F_{i}$

In Formula (2), $P$ suggests static pressure, $\tau_{i j}$ is stress vector, $\rho g_{i}$ denotes gravity component of fluid along i direction, and $F_{i}$ represent other energy items caused by resistance and energy.

The physical principle of energy conservation equation is the first law of thermodynamics, and its expression is as follows:

$$
\frac{\partial}{\partial t}(\rho h)+\frac{\partial}{\partial x_{i}}\left(\rho \mu_{i} h\right)=\frac{\partial}{\partial x_{i}}\left(k+k_{t}\right) \frac{\partial T}{\partial x_{i}}+S_{h}
$$

In Formula (3), $h$ is entropy, $k$ suggests molecular conductivity, and $k_{t}$ denotes other energy items caused by turbulent transport. When the velocity of fluid flow reaches the corresponding numerical value, the flow of fluid is changed from laminar to turbulent. Turbulence is a relatively complex flow [15]. Turbulence equation is needed to calculate turbulence. Two-equation model $k-\varepsilon$ is selected for calculation, and its expression is:

$k$ equation:

$\rho \frac{D k}{D t}=\frac{\partial}{\partial x_{i}}\left[\left(\mu+\frac{\mu_{t}}{\sigma_{k}}\right) \frac{\partial k}{\partial x_{i}}\right]+G_{k}+G_{b}-\rho \varepsilon$

$\varepsilon$ equation:

$\rho \frac{D \varepsilon}{D t}=\frac{\partial}{\partial x_{i}}\left[\left(\mu+\frac{\mu_{t}}{\sigma_{\varepsilon}}\right) \frac{\partial \varepsilon}{\partial x_{i}}\right]+C_{1 \varepsilon} \frac{\varepsilon}{k}\left(G_{k}+C_{3 \varepsilon} G_{b}\right)-C_{2 \varepsilon} \rho \frac{\varepsilon^{2}}{k}$

In Formulas (4) and (5), $\mu$ is laminar viscous coefficient, $\mu_{t}$ is turbulent viscous coefficient, $G_{k}$ is turbulent energy generated by laminar velocity gradient, $G_{b}$ is turbulent energy generated by buoyancy, and $C_{1 \varepsilon}, C_{2 \varepsilon}, C_{3 \varepsilon}, \sigma_{k}$, and $\sigma_{\varepsilon}$ are empirical coefficients. Their values are shown in Table 1.

The turbulent viscous coefficient can be expressed by the following formula:

$\mu_{t}=\rho C_{\mu} \frac{k^{2}}{\varepsilon}$

$C_{\mu}$ is a turbulence constant.

Table. $1 k-\varepsilon$ two-equation model coefficient

\begin{tabular}{|l|l|l|l|l|}
\hline$C_{1 \varepsilon}$ & $C_{2 \varepsilon}$ & $C_{\mu}$ & $\sigma_{k}$ & $\sigma_{\varepsilon}$ \\
\hline 1.44 & 1.92 & 0.99 & 1.0 & 1.3 \\
\hline
\end{tabular}




\subsection{Simplification and MNeshing of the Geometric Model of the Battery Box}

When equipment conditions permit, 1:1 model can be used for experiment, but there are many factors to be considered in CFD simulation calculation. Generally, no real geometric model based on actual battery box will be established for CFD simulation. Because of the complexity of the battery box structure model, it is difficult to complete the grid generation. Even if it is completed, it is difficult to ensure the accuracy of the grid, and the CFD simulation calculation with low grid accuracy has no computational value.

Assuming that the accuracy of grid generation is good, the number of grids and nodes will also make the simulation calculation too large and even more seriously, the calculation model will not be able to get the results. In the next work, it is necessary to simplify the geometric model of the primary battery box. By simplifying the geometric model of the battery box, the accuracy of CFD simulation calculation can be improved and the workload can be reduced.

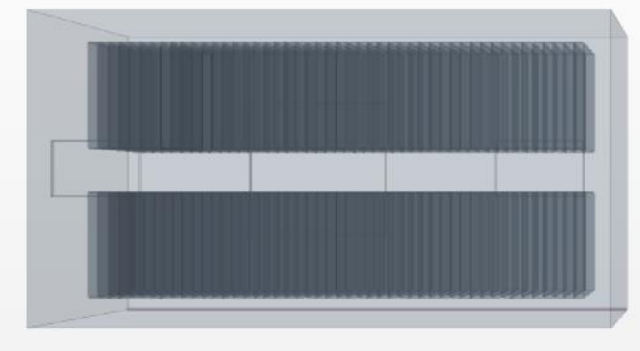

(a) Simplified battery box overlook

The following methods can be used to simplify the geometric model of battery box before grid generation.

Some minor components in the battery box, such as battery management system, conductors, side panels, etc., are small in size and have little influence on the whole calculation domain, and can be ignored.

The geometric model can be modified locally without affecting the flow field characteristics, and keep the regular structure as far as possible. The purpose is to reduce the difficulty of grid division and improve the speed of CFD simulation calculation. Symmetrical boundary or periodic boundary can be used for geometric structure with respect to plane or axisymmetric.

This method can be used in battery box modeling to reduce the computational load of CFD simulation and improve the computational speed of CFD simulation.

According to the above method, the threedimensional geometric model of battery box is simplified, and the simplified model is shown in Figure 2.

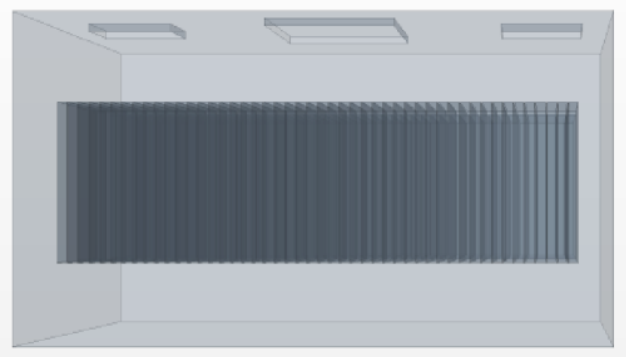

(b) Simplified battery box front view

Figure 2. The simplified model

In order to obtain high-precision numerical results, the key is to obtain high-quality grids. Grid division is an important part of the whole numerical simulation process. Grid generation takes up most of the time required for CFD simulation calculation. Improving the efficiency of grid generation is very important to save CFD simulation calculation time. At present, there are three main types of grid used in CFD simulation: structured grid, unstructured grid, and hybrid grid.

The adjacency of grid points in structured grid is orderly and regular. Except for boundary points, the number of adjacent grid points in internal grid points is the same (two in one dimension, four in two dimensions, and six in three dimensions).

The topological structure of structured grids is evenly distributed in the rectangular domain, and the grid nodes are all distributed on the grid line. Structured grid has simple data structure, small storage space, fast generation efficiency and high calculation accuracy, but there are also shortcomings.
Structured grid is only suitable for shape-regular graphics, and it is difficult to generate structured grid for complex geometric shapes.

With the rapid progress of science and technology, it is difficult for structured grids to fully adapt to geometric models with complex shapes.

In recent decades, many experts have made significant breakthroughs in grid generation technology and solved the difficulties faced by structured grids.

Unstructured grid means that the adjacency between grid nodes is disordered and irregular, and each grid point has a different number of adjacent grids. Unstructured grids have many advantages over structured grids.

They can be filled into every spatial location in the computational domain, ensure the accuracy of the initial position of the gridding model boundary, change the location of nodes in the grid, and delete or add nodes.

The way of unstructured grid generation also has some shortcomings. 
Because of its large number of nodes and the corresponding human processing, the storage space and computer performance are required to be high. Now, the improvement of computer performance can also make unstructured grid generation for complex geometries. However, the number of unstructured grid is too large to make simulation calculation take a lot of time.

Hybrid grid is the combination of structured grid and unstructured grid. It combines the advantages of structured grid and unstructured grid to make up for the shortcomings between them. Hybrid grid generation method uses structured grid generation in the near wall area of the model and unstructured grid generation in other areas.

This method solves the problem that many complex structures cannot be meshed, and has more credibility for the correlation analysis of complex structures. The heat dissipation model of the battery box studied here is simplified, and its shape is regular. The structure of the battery system can be regarded as a symmetrical structure, so half of the simplified geometric model can be meshed directly. Using STAR-CCM + software, grid generation of batteries and battery boxes is carried out. As shown in Figure 3, grid 1 is the grid generator selected for grid generation of batteries, and grid 2 is the grid generator selected for grid generation of battery box.

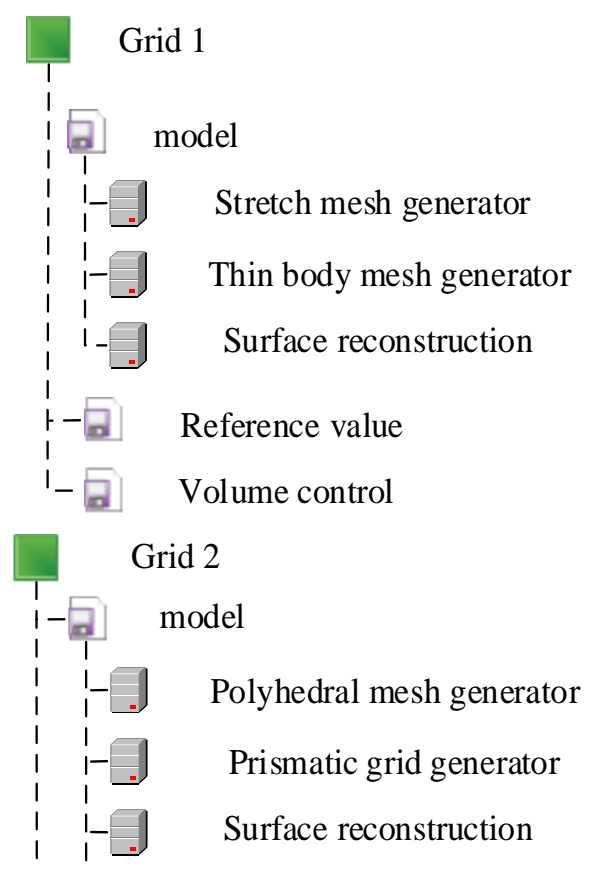

Figure 3. The setting of the grid model

A polyhedral grid generator is used to generate hexahedral mesh for the battery box. The prism layer grid generator is used in the corner part of the battery box and the position of the air inlet and outlet. After simplifying the lithium ion battery, the shape of the pole is a thin rectangular body.
The thin grid generator and the stretching grid generator are used for the battery box. The grid cell and battery simplified are shown in Figure 4.

The grid model of battery box is shown in Figure 4(a). It has a huge number of grids, about 10 million. The grid model of battery box and the grid model of lithium ion battery under the perspective state of Figure 4(b) can clearly see the grid of battery surface. The quality of battery box and battery grid generation is high, and the grid distribution is uniform. In the next CFD simulation calculation, a lot of computing time can be saved, and the reliability of the results will be guaranteed.

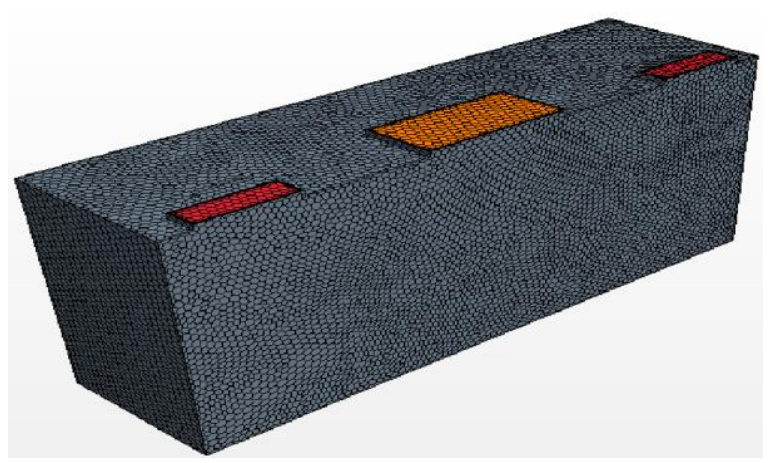

(a) Battery box grid model

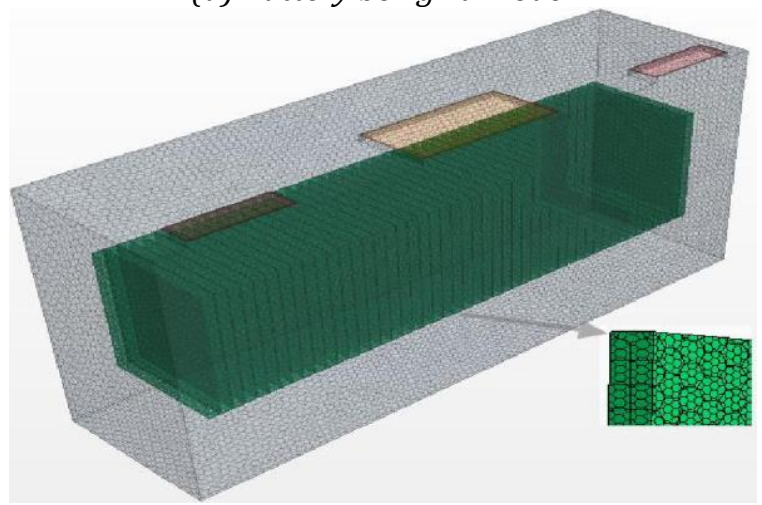

(b) Battery box grid model and lithium ion battery grid model in perspective

Figure 4. The grid model of battery box

\section{Research on Heat Dissipation of Lithium Ion Battery Box}

Lithium ion batteries have many advantages, making them the main force of power batteries in pure electric vehicles. However, lithium ion batteries generate a lot of heat in the working process, which will lead to the rise of battery temperature and seriously affect the performance of lithium ion batteries.

The CFD software STAR-CCM+ is set up to establish the fluid-solid coupling model, select the turbulence model and so on. STAR-CCM+ is used to solve the thermal effect of batteries by hydrodynamic method, and then the flow field and temperature field data of the calculation model of the battery box are analyzed and optimized. 


\subsection{CFD simulation calculation and analysis of original battery box}

The lithium ion battery box uses the forced air cooling method to heat the battery box.

The cold air passes through the intake ports 1 and 2 of the upper end of the battery box and enters the battery box to exchange heat for the heating battery unit.

The hot air is pumped out by the exhaust fan through the outlet of the battery box.

The actual temperature of the battery box will not be very high in the actual working situation, here. The physical properties of air (density $\rho$, specific heat $\mathrm{C}$, thermal conductivity $\lambda$, etc.) in the temperature range do not change much, and can be replaced by the physical properties of air at room temperature. Therefore, the fluid-structure coupling model in the computational fluid dynamics software STAR-CCM+ can be set as follows.

Physical model setting of fluid region:

a. Cooling air $300 \mathrm{~K}$, inlet wind speed $20 \mathrm{~m} / \mathrm{s}$;

b. Three-dimensional space model, implicit infinitive time model, gas physical property model and K-Epsilon turbulence model are selected;

c. Gas model chooses constant air and density.

$\mathrm{d}$. The position of import and export is free flow.

Physical model setting of solid region:

a. The initial temperature of the battery should be $300 \mathrm{~K}$.

b. The space model chooses three-dimensional kind, time model chooses implicit indefinite length, and physical property chooses solid;

c. The solid model chooses the batteries in the material library. According to the physical data given by the battery manufacturer, the density, thermal conductivity (anisotropy), and specific heat capacity of the batteries are set;

d. The volume heat source is selected for the battery heat source, and the data is set according to the calculation results in Chapter 2 .

e. The boundary condition of solid region is wall.

After setting up in the above way, STAR-CCM + software can simulate the calculation model of lithium ion battery box.

Taking the air-cooled heat dissipation system of the battery box of the pure electric bus as the research object, through the theoretical calculation of the heat generation rate of the power battery under the limit operating conditions of the pure electric bus, STAR-CCM + software is used to set the relevant data. The CFD simulation calculation of the battery box is carried out.

The CFD simulation calculation of the original battery box is shown in Figure 5 .

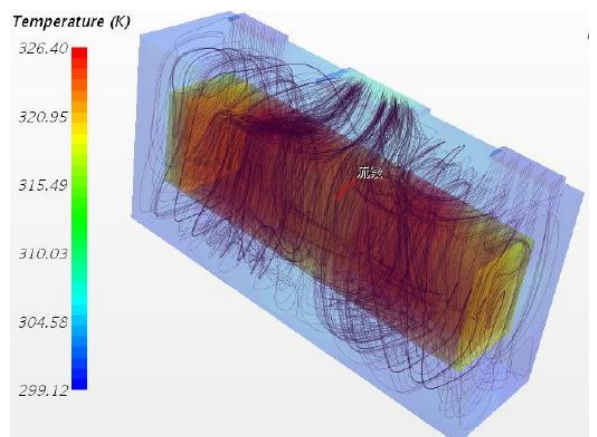

(a) Internal perspective temperature field and streamline diagram of the battery box

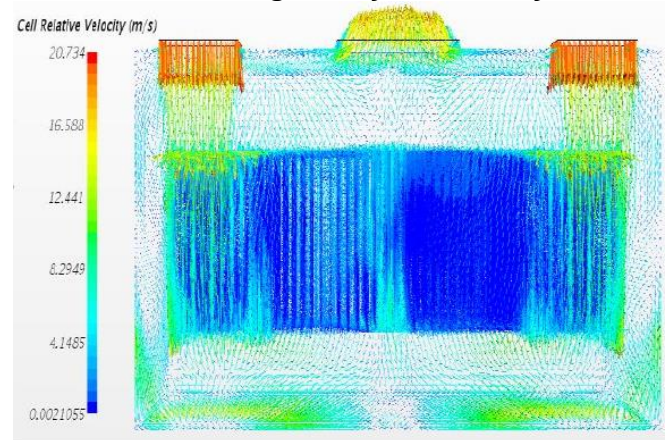

(b) Battery box internal velocity field

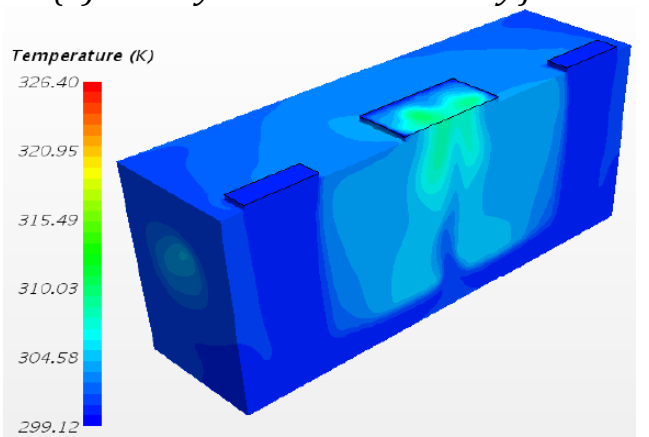

(c) Temperature field of the battery box Temperature $(K)$

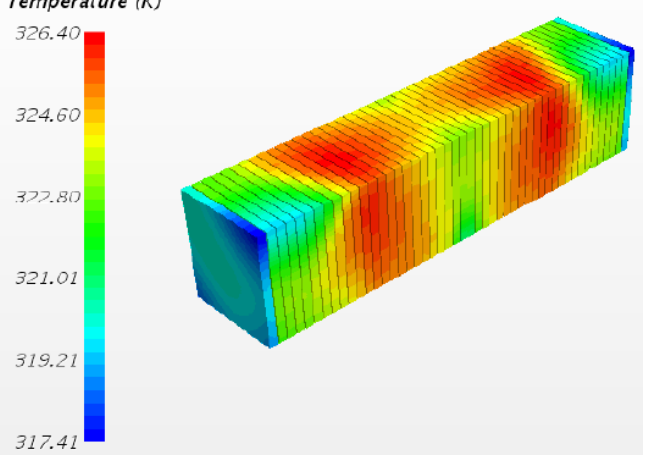

(d) Lithium ion battery temperature field

Figure 5. Battery box fluid dynamics simulation calculation chart

From Figure 8(a) and Figure 8(b), it can analyze that the air flow inside the battery box enters from the air inlet at both ends, and most of the air flow flows directly from the air outlet at the upper part of the battery box, which results in poor heat dissipation performance of the battery box. Figure 8(c) shows that the temperature of the gas in the air outlet of the battery box rises about $10 \mathrm{~K}$, indicating 
that the battery box has certain heat dissipation capacity. Figure $8(\mathrm{~d})$ shows that the maximum temperature of lithium ion batteries is $326.4 \mathrm{~K}$ $\left(53.3^{\circ} \mathrm{C}\right)$, the minimum temperature is $317.4 \mathrm{~K}$ $\left(44.3^{\circ} \mathrm{C}\right)$, and the temperature difference between batteries is $9.0 \mathrm{~K}$. If the pure electric bus is in this extreme condition for a long time or at high frequency, the heat dissipation capacity of the lithium ion battery box is limited, which has a certain impact on the battery life and consistency. As a result, the battery life between the outlet and the intake of the battery box is much lower than that of the lithium ion battery at the outlet and intake positions, thus affecting the overall performance of the pure electric bus.

\subsection{Orthogonal design}

Orthogonal experimental design method is to design the experimental scheme for different experimental factors and different levels of factors affecting the test results, and to find the best combination of the optimal design method to meet the test conditions through experimental analysis. The main steps of designing the orthogonal experimental scheme are: a. defining the experimental purpose and determining the experimental index; b. determining the factors to be investigated and selecting the appropriate level; c. selecting the appropriate orthogonal table; $d$. designing the table head; e. compiling the experimental scheme. In order to understand the definition of orthogonal experiment, it is necessary to know the following concepts: experimental factors are the factors that are investigated in the experiment (factors refer to the factors that may affect the experimental indicators); factor level refers to the different values taken by the factors in the various states of the experiment; and experimental indicators are the characteristic quantities used to measure the experimental results in an experiment.

After the orthogonal experiment design is completed, some methods are needed to analyze the experimental data. Range analysis and variance analysis are the analysis methods of orthogonal experiment adopted in the following. The characteristics of range analysis method are easy to understand, intuitive image and strong contrast of results. Through simple calculation and judgment, the primary and secondary factors, optimal level, optimal collocation, and optimal combination scheme of experimental optimization can be obtained. The magnitude of the range reflects the influence of the corresponding experimental factors on the experimental results. The larger the range is, the greater the impact on the experimental results is, and the more important the factor is. On the contrary, the smaller the influence on the experimental results is, and the less important the factor is. However, range analysis cannot verify the test error. If the test error is larger, it will affect the acquisition of the optimal scheme of orthogonal test.

Variance analysis can solve the problems that range analysis cannot solve, mainly including: estimating the error of experiment and analyzing the influence of experiment error; judging the significance and order of experiment interaction and factors; neglecting the interaction that has no significant effect or acting as an error analysis item; giving the reliability of the optimal scheme and determining the confidence interval of the optimal scheme.

The process of variance analysis is as follows: a. First, the deviation squares and the corresponding degrees of freedom of each factor level are calculated from the test data, and then the estimates of variance of different factors level of each test index are calculated; $b$. The estimates of variance of test error are calculated; c. The FA values are calculated to determine the significance; $d$. the process and results of variance analysis are tabulated.

The sum of squares of column deviations is the sum of squares of deviations between the average and the total average of the corresponding test indices at each level of $\mathrm{j}$-th column. The formula is as follows:

$S_{j}=\frac{b}{a} \sum_{i=1}^{b} K_{i j}{ }^{2}-\frac{1}{a}\left(\sum_{i=1}^{q} K_{i}\right)^{2}$

In Formula (7): $b$ is the horizontal number of each test factor; $a$ is the test number; $i$ is the row number of the orthogonal test; $j$ is the column number of the orthogonal test. The degree of freedom of the sum of total deviation squares is equal to the test number minus 1 , and the degree of freedom of the sum of $j$-th column deviation square is equal to the number of levels of the column minus 1. In orthogonal test saliency detection, the detection method $F_{A}$ is usually used. In the specific implementation process, further calculation is needed to list the saliency level $\alpha$.

The above calculation can be processed by the specialized processing software of orthogonal test. $F_{A}$ is called $\mathrm{F}$ ratio of factor A, and the formula for calculating $F_{A}$ is as follows:

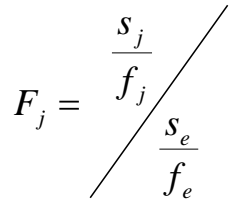

The sum of squares of experimental errors is equal to the sum of squares of deviations of all empty columns in the orthogonal table. It is called the sum of squares of random errors. Its formula is as follows:

$$
S_{e}=\sum_{c_{k}} S_{j}
$$


Power lithium ion batteries generate more heat when they work under the extreme operating conditions of pure electric bus. Battery boxes are needed to discharge the heat generated by power lithium ion batteries through the outlet of the battery box. Through CFD simulation calculation and analysis of the original battery box, most of the cold air entering the battery box from the intake port flows out directly from the upper part of the battery box by the outlet of the battery box, and the cold air entering the battery box is not fully utilized, which results in the temperature rise of the battery box and the temperature consistency between the battery cells cannot meet the requirements.

The above situation occurs for a long time, seriously affecting the power battery life.

In order to improve the heat dissipation performance of the original battery box, the corresponding improvement measures are put forward: a. By adjusting the air velocity of the intake port of the battery box, the air temperature entering the battery box through the intake port is changed; $b$. In structure, the size of the flow passage between the batteries in the battery box is changed; c. The guide plate is added inside the battery box.

Through the above three measures, the air temperature at the intake of the battery box and the flow field inside the battery box re changed, and the heat dissipation performance of the battery box is improved. The inner guide plate of the battery box is divided into upper guide plate (blue) and vertical guide plate (grey).

The specific structure of the guide plate is shown in Figure 5(a).

The guide plate is symmetrically distributed in the improved battery box, and its actual distribution in the improved battery box is shown in Figure 6 (b) and (c).

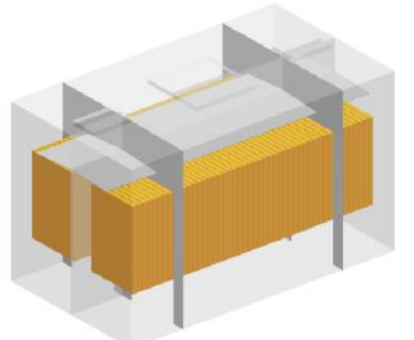

(a) Isometric side view of the deflector at a specific location within the battery compartment

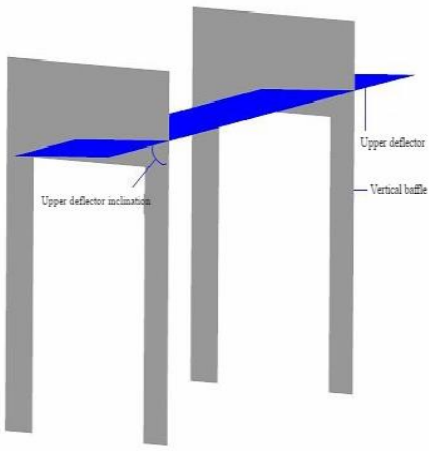

(b)Internal baffle added to the battery box

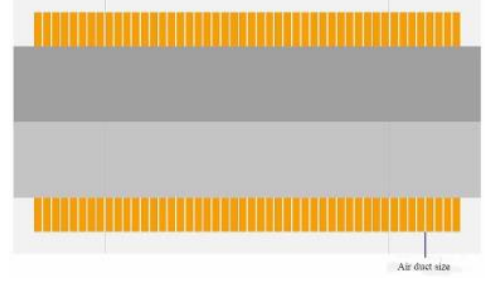

(c)Top view of the deflector in a specific position inside the battery box

Figure 6: The structure of the battery box deflector and its position in the battery box

\subsection{CFD simulation calculation and analysis of battery box with optimal solution}

Using STAR-CCM+ software, the CFD simulation calculation of the battery case is carried out. The CFD simulation calculation figure of the optimal scheme is shown in Figure 7 below. Compared with the streamlines in Figure 5(a), the streamlines in Figure 7 (a) are more evenly distributed in the battery box. The streamlines in the upper intake area of the battery box are obviously sparse, and the streamlines in the bottom area of the battery box are increased. This shows that the guide plate in the battery box plays a better role in guiding current.

Figure 7(b), compared with Figure 5(b), the air velocity in the cooling duct between the cells is $4 \mathrm{sm}$, and the air velocity around the bottom of the cell box is more stable and basically $8 \mathrm{sm}$, and there is no air velocity above $12 \mathrm{sm}$. The temperature distribution of lithium ion battery is more consistent, and the temperature difference is much lower in Figure 7 (c) compared with Figure 5(d). From Figure 7(c), it can be seen that the maximum temperature of lithium ion battery is $316.4 \mathrm{~K}\left(43.3^{\circ} \mathrm{C}\right)$, the minimum temperature is $314.1 \mathrm{~K}\left(41.0^{\circ} \mathrm{C}\right)$, and the temperature difference between batteries is $2.3 \mathrm{~K}$.

The fourth position is the highest working temperature area in the battery box, and the twentysecond position is the lower working temperature area in the battery box. By observing their internal temperature field, the consistency degree of the single battery is seen. From Figure 10 (d) and (e), it is possible to see the temperature distribution of the single battery in the battery box. The difference between the temperature distribution inside the cell is small, and the battery has good consistency.

Compared with the original battery box, the maximum temperature of the single battery of the optimized battery box is reduced by $3.06 \%$, and the temperature difference between the single battery is reduced by $74.44 \%$. CFD simulation results show that the heat dissipation performance of the battery box can be improved by adjusting the air velocity and temperature in the intake port of the battery box and changing the direction of air flow in the battery box. 


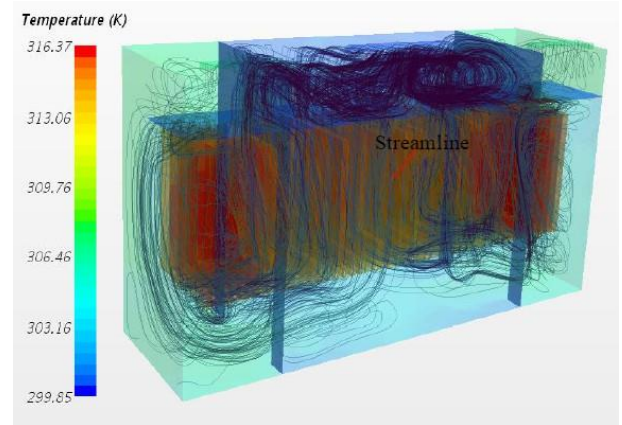

(a) The internal perspective temperature field and streamline diagram of the battery case of the

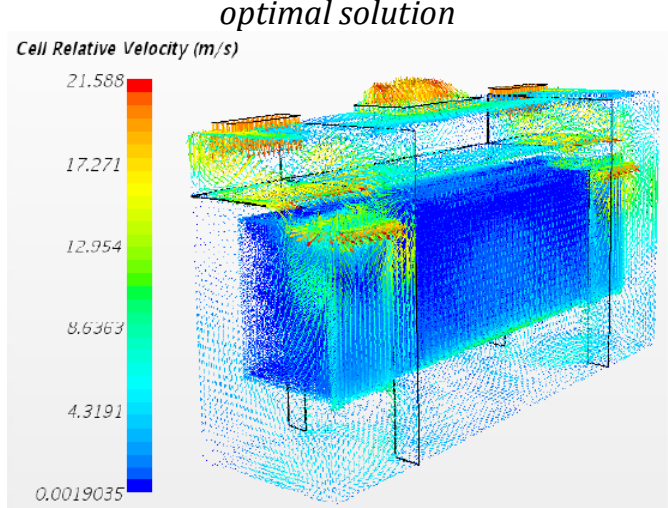

(b) Optimal tank interior velocity field

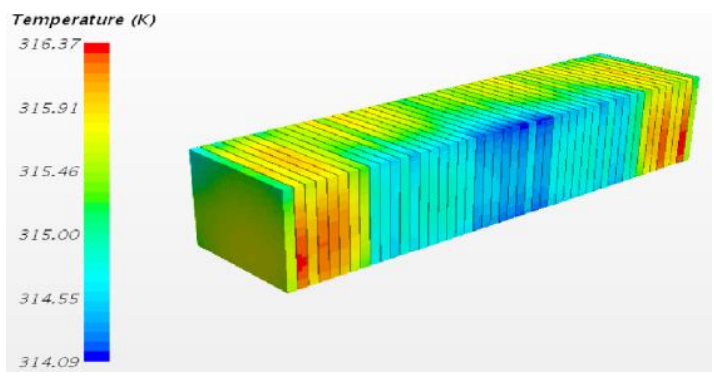

(c) Lithium ion battery temperature field

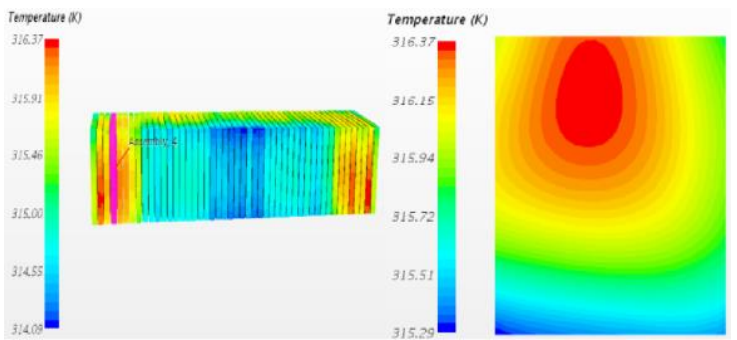

(d)Four-position single cell temperature field

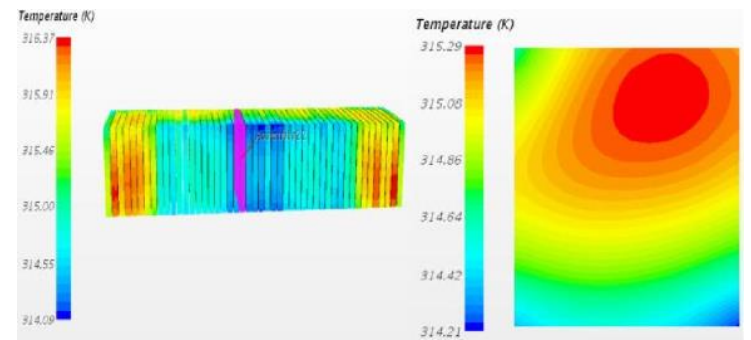

(d)Twenty-two single cell temperature field

Figure 7. Optimal solution of battery box flow line and temperature field, velocity field map

\subsection{Temperature field analysis of lithium ion battery}

Because of the large number of battery units, including 96 battery units, if the details of each cell are taken into account, it will consume a lot of energy to strictly deal with the details of the cell, and the complexity of iterative simulation will be very complex.

Therefore, each battery is regarded as a whole, and the internal structure is ignored. The geometric model of the battery pack is shown in Figure 8.

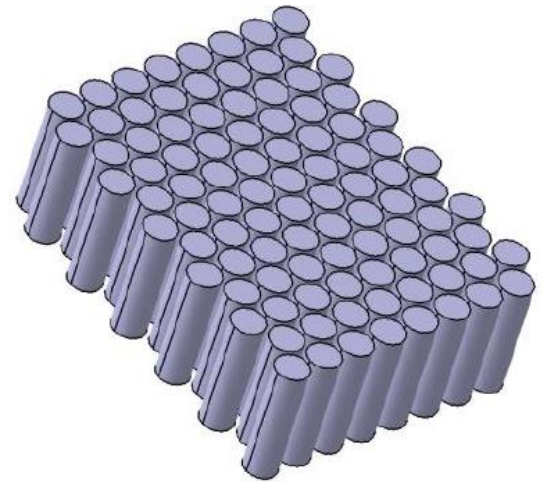

Figure 8. Battery pack structure

The shape size of the battery support structure is $65^{*} 190 * 215 \mathrm{~mm}^{3}$, the wall thickness of both sides is $9 \mathrm{~mm}$, and the wall thickness of the upper and lower sides is $7.5 \mathrm{~mm}$. Polycarbonate PC plastics are used.

The density is $1200 \mathrm{~kg} / \mathrm{m}^{3}$, the specific heat capacity is $1170 \mathrm{~J} / \mathrm{kg} \cdot \mathrm{K}$, and the thermal conductivity is $0.24 \mathrm{~W} / \mathrm{m} \cdot \mathrm{K}$.

The geometric model of the battery pack bracket is shown in Figure 9.

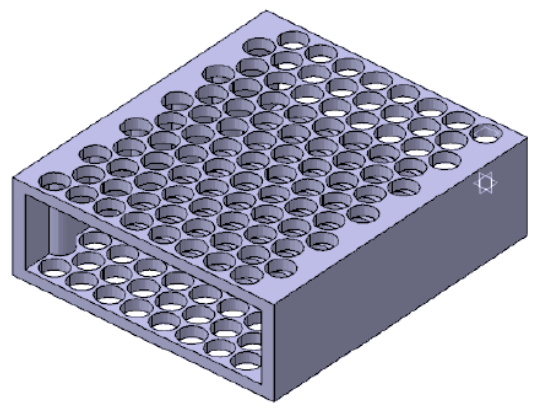

Figure 9. Battery holder structure

Considering that air is a bad conductor of heat and has poor heat dissipation effect under extreme conditions, liquid is chosen as heat transfer medium here.

The results show that the heat transfer coefficient of liquid is much higher than that of air. In order to achieve better heat dissipation effect, the whole battery pack and its support are completely immersed in liquid in the heat dissipation structure designed. 
A rectangular fluid field is drawn around the battery pack. The gap between the upper and lower fluid fields is $2.5 \mathrm{~mm}$ from the end of the battery.

The thickness of the two sides is $30 \mathrm{~mm}$.

The shape of the whole fluid field is $70 * 190 * 275 \mathrm{~mm}^{3}$.

The assembly structure of battery pack, bracket and fluid field is shown in Figure 10.
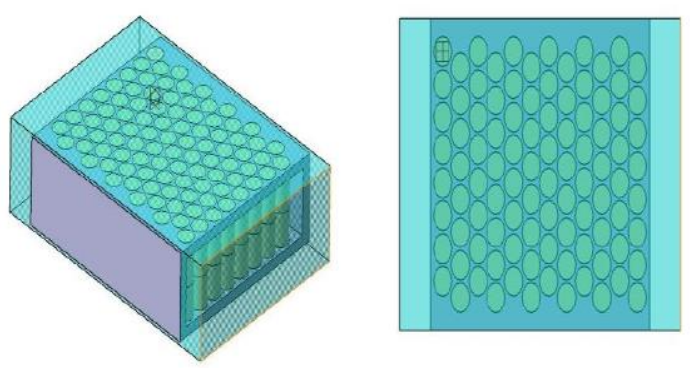

Figure 10. Battery pack and surrounding watershed structure

Because of the direct contact between heat transfer liquid and battery, in order to achieve insulation effect, non-conductive liquid must be selected. ATF hydraulic oil is selected as heat transfer medium to simulate and analyze the heat diffusion effect of batteries. The physical parameters of ATF hydraulic oil are as follows: density $843 \mathrm{~kg} / \mathrm{m}^{3}$; specific heat capacity $1885 \mathrm{~J} / \mathrm{kg} \cdot \mathrm{K}$; thermal conductivity $0.125 \mathrm{~W} / \mathrm{m} \cdot \mathrm{k} ;$ dynamic viscosity coefficient $0.0285 \mathrm{~kg} / \mathrm{m} \cdot \mathrm{s}$.

There are two ways to build the geometric model of battery pack: a. Using CAD software such as CATIA, AUTOCAD, and UG to build the geometric model of battery pack, and importing the established model into the Fluent Flow module; b. Using the Fluent module of ANSYS Workbench directly, the geometric model is built in the pre-processor (Geometry sub-module). The geometric model is built in CATIA software and imported into FlentFlow (Fluent) module. The grid sub-module is used to mesh the geometric model.

Due to the limited computational level of fluid dynamics simulation, it is difficult to solve the complex geometric model accurately. The main manifestation is that it is difficult to guarantee the meshing quality of complex geometric models, and there are great limitations in the meshing of details. Too many details will lead to serious distortion of grids and calculation distortion. In addition, a large number of grids need to be generated with high accuracy. Too many grids will result in too long iteration time, too much space and increased calculation cost. Therefore, the geometric model does not fully reflect the detailed structure of the cooling system of the battery pack. It is simplified to ensure that the main characteristics of the flow field are reflected, which reduces the complexity of the model and ensures the accuracy of the simulation calculation.
Based on the geometric model of battery pack and flow field, the computational domain is meshed and the finite element model is generated. There are two types of fluid field grids: structured grids and unstructured grids. Structured grids can be expressed by mathematical analytic formulas to generate grid data. They are sequential in data structure and they are easy to set up and have less computational load. They can control the encryption requirements in any direction manually and obtain high-quality meshing, but they are not suitable for geometric models with complex shapes. Unstructured grids have good adaptability to the shape of geometric bodies. They are suitable for complex geometric models. They can automatically select suitable topological structures and element types according to the shape changes. However, the amount of grids generated by unstructured grids is large, the quality of grids is not as good as that of structured grids, and the simulation iteration time is long.

The whole battery cell is meshed, including cell monomer and fluid domain. Different meshing methods are needed to mesh geometric bodies with different physical states. Two methods are used to mesh cell and fluid domain. The geometric model of battery selected is simple in shape and suitable for structured grid. It cannot only ensure the calculation accuracy, but also reduce the simulation iteration time. However, the geometric models of fluid domain and battery support are relatively complex, and unstructured grids are suitable for use, and the quality of grids is high.

Because of the symmetrical structure of the battery pack and the influence of the computer performance, in order to simplify the calculation and reduce the calculation time, the symmetrical surface is selected to simulate only the lower half of the battery pack, and the calculated results are still representative. The results of meshing are shown in Figure 11.

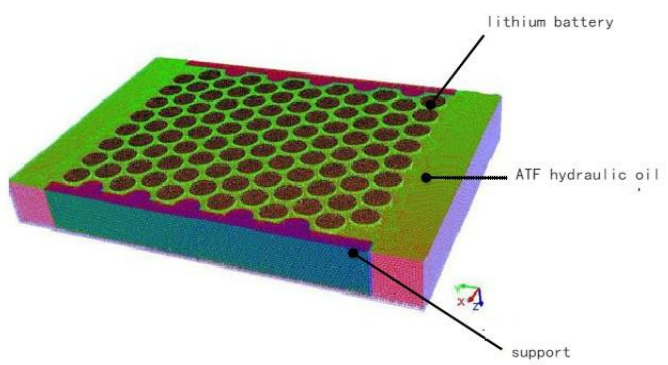

Figure 11. Battery pack and watershed grid

\section{Conclusion}

Based on the LCK6108EV lithium ion power battery box and power battery of Zhongtong bus, the heating mechanism of lithium ion batteries and the formula of the heating rate of batteries are firstly studied, and the estimation model of the heating rate of 
lithium-ion batteries is simplified. In order to improve the heat dissipation performance of the battery box, measures such as adding a guide plate in the battery box and changing the size of the air duct between the batteries are put forward.

The numerical simulation calculation of the battery box is carried out by using STAR-CCM+ simulation software, and the optimization design of the battery box is carried out according to the simulation results, and the optimum scheme of the heat dissipation of the battery box is determined.

The main work is as follows:

Firstly, by consulting a large number of domestic and foreign literatures on battery heat dissipation analysis and orthogonal experimental design, the development history of pure electric vehicle battery and the research progress of battery technology are comprehensively understood, and the theoretical knowledge of orthogonal experimental design is systematically sorted out.

Secondly, the heat generation mechanism of common lithium ion power batteries is deeply analyzed, and the heat calculation formula of the batteries is obtained. By analyzing the threedimensional thermal model of lithium ion batteries, Bernardi heat generation rate model is combined with the traditional automobile power balance equation to simplify the calculation model of the heat generation rate of lithium ion batteries.

Thirdly, by comparing the characteristics of the heat dissipation modes of the battery pack, the forced air cooling mode is selected as the heat dissipation mode of the battery box, and then the three-dimensional geometric model of the battery box is established by using three-dimensional software, and then simplified. The computational flow of CFD software and the types of finite element meshes are introduced. Then, the simplified geometric models of three-dimensional battery box and lithium ion battery are divided into hexahedral meshes using STAR-CCM+ software.

Fourthly, when the power battery is working under the limit condition of pure electric bus, CFD simulation analysis is carried out. The maximum temperature of lithium-ion battery is $326.4 \mathrm{~K}$ $\left(53.3^{\circ} \mathrm{C}\right)$, the minimum temperature is $317.4 \mathrm{~K}$ $\left(44.3^{\circ} \mathrm{C}\right)$, and the temperature difference between batteries is $9.0 \mathrm{~K}$, which will affect the service life of the battery. Improvement measures are put forward and orthogonal test method is adopted to reduce the number of tests. Through range analysis and variance analysis of multi-index test data of orthogonal experiment, the factors or levels that have the greatest impact on each test index are determined.

Then, two optimal schemes are obtained by comprehensive balance method. Finally, the two optimal schemes are simulated and calculated, and the optimal scheme is obtained by data comparison. The simulation results of the optimal scheme show that the maximum temperature of lithium ion batteries is $316.4 \mathrm{~K}\left(43.3^{\circ} \mathrm{C}\right)$, the minimum temperature is $314.1 \mathrm{~K}\left(41.0^{\circ} \mathrm{C}\right)$, and the temperature difference between batteries is $2.3 \mathrm{~K}$. Compared with the lithium ion battery in the original battery box, the maximum temperature of the single battery is reduced by $3.06 \%$, and the temperature difference between the single batteries is reduced by $74.44 \%$.

Adjusting the flow rate and temperature of the air in the intake port of the battery box and changing the direction of the air flow in the battery box can improve the heat dissipation performance of the battery box.

\section{Acknowledgement}

Research startup subject of Yangtze Normal Universi ty: 2017KYQD16

\section{References}

[1] Hao, Y., Dong, X. Y., Deng, Y. X., Li, L. X., \& Ma, Y. (2016). What influences personal purchases of new energy vehicles in china? an empirical study based on a survey of Chinese citizens. Journal of Renewable \& Sustainable Energy, 8(6), 262-269.

[2] Liu, X., Yan, H. J., Tian, N., \& Zhao, G. Q. (2017). Cfd simulation analysis and research based on engine air intake system of automotive. Proceedings of the Spie, 322, 103224G.

[3] He, Y., Zhou, W., Li, M., Ma, C., \& Chen, Z. (2016). An adaptive fuzzy logic-based energy management strategy on battery/ultracapacitor hybrid electric vehicles. IEEE Transactions on Transportation Electrification, 2(3), 300-311.

[4] Xu, F., Jiao, X., Wang, Y., \& Yuan, J. (2017). Battery-lifetime-conscious energy management strategy based on sp-sdp for commuter plug-in hybrid electric vehicles. Ieej Transactions on Electrical \& Electronic Engineering, 13(3), 472479.

[5] Hannan, M. A., Hoque, M. M., Hussain, A., Yusof, Y., \& Ker, P. J. (2018). State-of-the-art and energy management system of lithium-ion batteries in electric vehicle applications: issues and recommendations. IEEE Access, 6(99), 1936219378.

[6] Chang, T. B., Sheu, J. J., Huang, J. W., Lin, Y. S., \& Chang, C. C. (2018). Development of a cfd model for simulating vehicle cabin indoor air quality. Transportation Research Part D Transport \& Environment, 62, 433-440.

[7] Qiao, Z., Deng, W., Zhang, S., \& Jian, W. (2016). A rule based energy management system of experimental battery/supercapacitor hybrid energy storage system for electric vehicles. Journal of Control Science and Engineering, 2016, (2016-7-5), 2016, 4. 
[8] Zhang, S., Rui, X., \& Cao, J. (2016). Battery durability and longevity based power management for plug-in hybrid electric vehicle with hybrid energy storage system. Applied Energy, 179, 316-328.

[9] Hong, J., Wang, Z., \& Peng, L. (2016). Voltage fault precaution and safety management of lithium-ion batteries based on entropy for electric vehicles. Energy Procedia, 104, 44-49.

[10] Wang, L., Wang, L., Liao, C., \& Zhang, W. (2018). Research on multi-parameter evaluation of electric vehicle power battery consistency based on principal component analysis, 23(5), 711-720.

[11] Zhao, J., Rao, Z., Huo, Y., Liu, X., \& Li, Y. (2017). Corrigendum to "thermal management of cylindrical power battery module for extending the life of new energy electric vehicles" [appl. thermal eng. 85 (2015) 33-43]. Applied Thermal Engineering, 122.
[12] Rizoug, N., Mesbahi, T., Sadoun, R., Bartholomeüs, P., \& Moigne, P. L. (2018). Development of new improved energy management strategies for electric vehicle battery/supercapacitor hybrid energy storage system. Energy Efficiency, 11(5), 1-21.

[13] Tong, S., Fung, T., Klein, M. P., Weisbach, D. A., \& Park, J. W. (2017). Demonstration of reusing electric vehicle battery for solar energy storage and demand side management. Journal of Energy Storage, 11, 200-210.

[14] Trovao, J. P., Roux, M. A., Menard, E., \& Dubois, M. (2017). Energy- and power-split management of dual energy storage system for a three-wheel electric vehicle. IEEE Transactions on Vehicular Technology, (99), 1-1.

[15] Cao, Y., Wang, N., Kamel, G., \& Kim, Y. J. (2016). An electric vehicle charging management scheme based on publish/subscribe communication framework. IEEE Systems Journal, (99), 1-14. 